\title{
Effect of Spin-Torque on Thermal Mag-Noise in a TMR Read Head
}

\author{
Yasushi Endo, Tetsuya Abe, Mitsuru Suzuki, and Masahiro Yamaguchi
}

ECEI, Grad. Sch. of Eng., Tohoku Univ., 6-6-05 Aoba, Aramaki, Aoba-ku, Sendai, 980-8579, Japan

This paper describes the noise spectra in the GHz range of tunneling magnetoresistive (TMR) read heads as a function of both bias current and external magnetic field in order to understand the effect of spin-torque (ST) on the thermally excited magnetization fluctuation noise (thermal mag-noise) in the TMR heads. A strong noise spectral peak at around 3-4 GHz is either enhanced or reduced depending on both the bias current and the external magnetic field. This result demonstrates that the ST influences the thermal mag-noise originated from the free layer under the positive (or negative) bias current in case the magnetization of the free and reference layers are nearly anti-parallel (or parallel) to each other.

Key words: noise spectrum, GHz range, thermal mag-noise, spin-torque, TMR heads

\section{Introduction}

The continued increase in the areal density of magnetic recording media required further smaller read head down to $30 \mathrm{~nm}$ and below. Accordingly, the signal-to-noise ratio (SNR) of read-out data from a current-perpendicular-to-plane (CPP) type tunneling magnetoresistive (TMR) ${ }^{1}$ ) and giant-magnetoresistive (GMR) spin-valve ${ }^{2,3)}$ heads would be markedly degraded by the thermally excited magnetization fluctuation noise (thermal mag-noise) ${ }^{4-6}$ ) and the non-thermal fluctuation noise originated from the spin-torque (ST) generated by the spin polarized current through the CPP stack $^{7-11)}$ as well.

Therefore, this paper investigates noise spectra in the $\mathrm{GHz}$ range of TMR read heads as a function of both dc bias current and external magnetic field in order to understand the effect of ST on the thermal mag-noise in the TMR heads.

\section{Experimental Procedure}

A TMR read head bars were composed of around 50 heads. The number of measured heads was 15 . The physical sensor width was $100 \mathrm{~nm}$ and the strip height was $100 \mathrm{~nm}$. The magnetization of the free layer, $\left(M_{\mathrm{s}}\right)_{\mathrm{FL}} t_{\mathrm{FL}}$, is approximately $5.0 \times 10^{-4} \mathrm{emu} / \mathrm{cm}^{2}$. The resistance-area product of the tunnel barrier, $R A$, is approximately $4 \Omega \cdot \mu \mathrm{m}^{2}$. The dc bias current up to \pm 1.8 $\mathrm{mA}$ was injected perpendicularly to the head plane. The external magnetic field up to $\pm 100 \mathrm{kA} / \mathrm{m}$ was applied in parallel to the head plane. The "positive" direction of bias current is defined in this paper as flowing from a free layer to a reference layer. Similarly the "positive" direction of external magnetic field is defined as being anti-parallel to that of magnetization in the reference layer. The noise spectra of TMR heads were measured in the frequency range of $0.1-10 \mathrm{GHz}$ using a spectrum analyzer through a $36 \mathrm{~dB}$ low noise amplifier.

\section{Results and discussion}

The noise spectra for any of the measured TMR heads depend on both the bias current and the external magnetic field. Figure 1 shows the typical noise spectrum in TMR heads as a function of external magnetic field with applied dc bias current of $\pm 1.2 \mathrm{~mA}$. In the case of the head \#1[Fig. 1(a)], at the bias current of $+1.2 \mathrm{~mA}$ and without the external magnetic field, a main peak is clearly observed at approximately $4.0 \mathrm{GHz}$. The peak once shifts toward lower frequency side with increasing external magnetic field up to approximately $+39.8 \mathrm{kA} / \mathrm{m}$, and then reverses to higher frequency side in the field intensity range between +55.7 and +79.6 $\mathrm{kA} / \mathrm{m}$. The peak becomes sharper and its intensity is enhanced with increasing external magnetic field. A (a) Bias Current $=+1.2 \mathrm{~mA}$

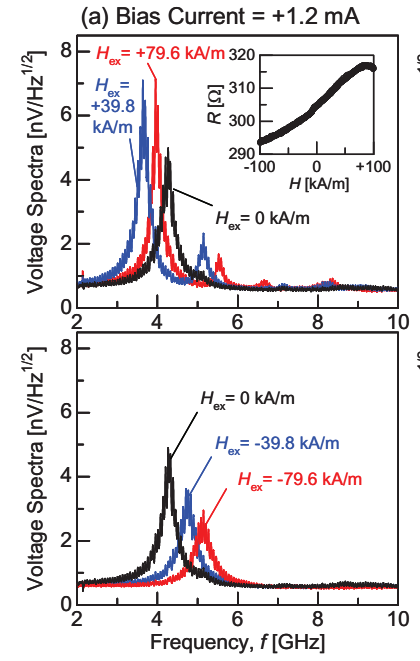

(b) Bias Current $=-1.2 \mathrm{~mA}$

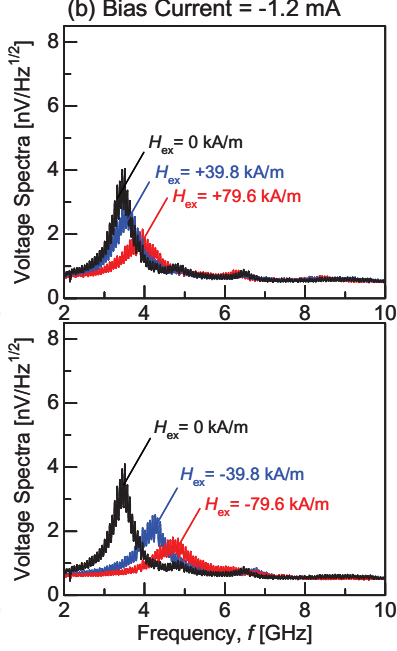

Fig. 1 Noise spectrum in the TMR head (\#1) as a function of external magnetic field $\left(H_{\mathrm{ex}}\right)$ with the dc bias current of (a) $+1.2 \mathrm{~mA}$ and (b) $-1.2 \mathrm{~mA}$. The inset in Fig. 1(a) represents the curve of the resistance $(R)$ versus the external magnetic field $\left(H_{\mathrm{ex}}\right)$ in the head $\# 1$ at the dc bias current of $+1.2 \mathrm{~mA}$. 
weak peak is also observed at approximately $5.0 \mathrm{GHz}$, and monotonically shifts toward the high frequency side with increasing external magnetic field. Its peak intensity is once enhanced with increasing external magnetic field up to approximately $+39.8 \mathrm{kA} / \mathrm{m}$, and then lowers in the field intensity range between +55.7 and $+79.6 \mathrm{kA} / \mathrm{m}$. Other weak peaks are seen above 6.0 $\mathrm{GHz}$ only at the external magnetic field more than $+55.7 \mathrm{kA} / \mathrm{m}$. As was reported in ref. 9), the thermal mag-noise depends on only the intensity of external magnetic field, which always suppresses the peak intensity and shifts the peak toward higher frequency side. The spin-torque (ST) enhancement of thermal mag-noise is observed depending on both the direction of the dc bias current and that of the external magnetic field. Therefore, both the main peak at around $4.0 \mathrm{GHz}$ and the weak peak at around $5.0 \mathrm{GHz}$ are mainly attributed to the thermal mag-noise originated from the free layer. Their peak intensity strengthens with increasing external magnetic field only under the combination of "positive" bias current and the "positive" magnetic field. This feature corresponds to that reported in ref. 9). These results also suggest that the thermal mag-noises are influenced by the ST when applied the positive bias current and positive magnetic field and that the magnetization of the free and reference layers are nearly anti-parallel $(A P)$ to each other. Other weak peaks above $6.0 \mathrm{GHz}$ may be caused by the noise originated from the non-uniform magnetization fluctuation at the edge of the free layer, as was discussed in ref. 12).

On the other hand, at the bias current of $-1.2 \mathrm{~mA}[\mathrm{Fig}$. $1(\mathrm{~b})$, the main peak is clearly observed at approximately $3.5 \mathrm{GHz}$ without the external magnetic field. The peak shifts toward the high frequency side and its intensity lowers as the intensity of external magnetic field increases regardless of the field direction. This peak is mainly attributed to the thermal mag-noise originated from the free layer, as described above. These noise spectra indicate that the effect of ST on the thermal mag-noise disappears under the negative bias current in case the magnetization of the free and reference layers are nearly parallel $(P)$ to each other. The reason for this might be that the spin polarization decreases when the bias current direction is reversed.

In the case of the head $\# 2$, at the bias current of +1.2 $\mathrm{mA}$ [Fig. 2(a)] and without the external magnetic field, a main peak is clearly observed at approximately $3.0 \mathrm{GHz}$. The peak slightly shifts toward the high frequency side as the intensity of external magnetic field increases regardless of the field direction. Additionally, the peak intensity is enhanced with increasing external magnetic field up to approximately $+39.8 \mathrm{kA} / \mathrm{m}$, and then reduced in the field intensity range between +55.7 and +79.6 $\mathrm{kA} / \mathrm{m}$. A weak peak is also observed at approximately 4.2 GHz. The peak shifts toward the high frequency side and its intensity lowers with increasing external magnetic field intensity, and then disappears at approximately $\pm 55.7 \mathrm{kA} / \mathrm{m}$. Other weak peaks are seen above $6.0 \mathrm{GHz}$ in all external magnetic field range. On the other hand, as can be seen in Fig. 2(b), an opposite (a) Bias Current $=+1.2 \mathrm{~mA}$

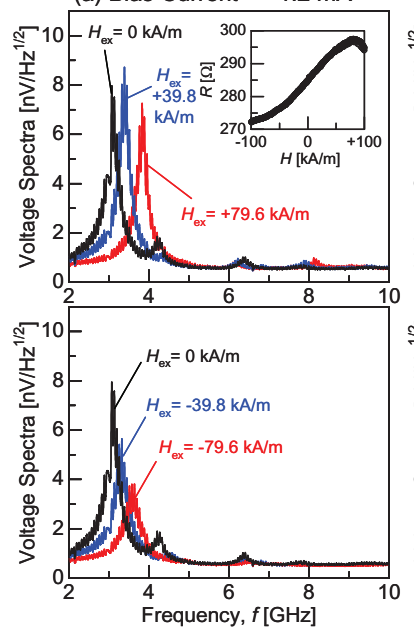

(b) Bias Current $=-1.2 \mathrm{~mA}$

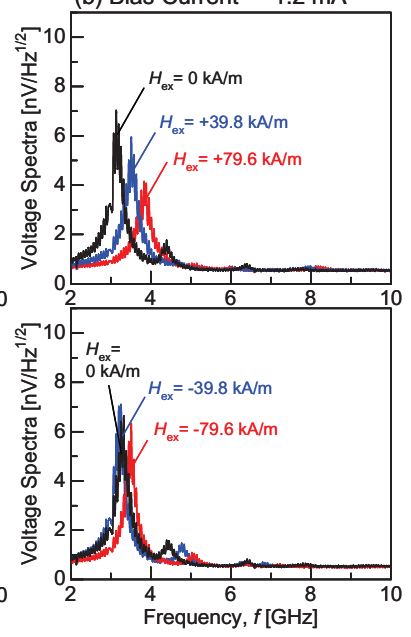

Fig. 2 Noise spectrum in the TMR head (\#2) as a function of external magnetic field $\left(H_{\mathrm{ex}}\right)$ with the dc bias current of (a) $+1.2 \mathrm{~mA}$ and (b) $-1.2 \mathrm{~mA}$. The inset in Fig. 2(a) represents the curve of the resistance $(R)$ versus the external magnetic field $\left(H_{\mathrm{ex}}\right)$ in the head \#2 at the dc bias current of $+1.2 \mathrm{~mA}$.

trend is observed when the bias current direction is reversed. Thus, changes in the noise spectrum with the magnetic field are quite different from those of the head \#1. From these results, it is suggested that the main peak are attributed to the thermal mag-noise enhanced by the ST under a positive (or negative) bias current in the positive (or negative) field range, namely in the case of nearly $A P$ (or $P$ ) state. It is also revealed that other weak peaks are ascribed to either only the thermal mag-noise or the noise originated from the non-uniform magnetization fluctuation at the edge of the free layer.

In contrast to the field dependence of the noise spectrum, the change in noise spectrum with bias current is quite different, revealing a large variation of peak intensity with little frequency shift, as can be noticed in Fig. 3. In the case of the head \#1[Fig. 3(a-1)], at the external magnetic field of $+79.6 \mathrm{kA} / \mathrm{m}$, which results in the nearly $A P$ state (indicated by the inset $R$ - $H$ curve of Fig. 1(a)), a main peak at around $4.0 \mathrm{GHz}$ becomes sharper and its intensity is markedly enhanced when the bias current direction is changed from negative to positive and its amplitude is increased. These results indicate that the noise spectra are influenced by the spin-torque (ST). On the other hand, as shown in Fig. 3(a-2), at the external magnetic field of $-79.6 \mathrm{kA} / \mathrm{m}$ which results in the nearly $P$ state, a main peak at around $5.0 \mathrm{GHz}$ slightly becomes broad and its intensity is slowly enhanced when the bias current direction is changed from positive to negative and its amplitude is increased. These results mean the effect of only the thermal mag-noise on the noise spectrum. Thus, the influence of the ST on the thermal mag-noise is not observed under the negative bias current in the $P$ state. This behavior is similar to the asymmetry of head noise in CPP heads for the bias current, as was reported in ref. 10). The reason for this might be that the spin 
(a) (a-1) $H_{\mathrm{ex}}=+79.6 \mathrm{kA} / \mathrm{m}$

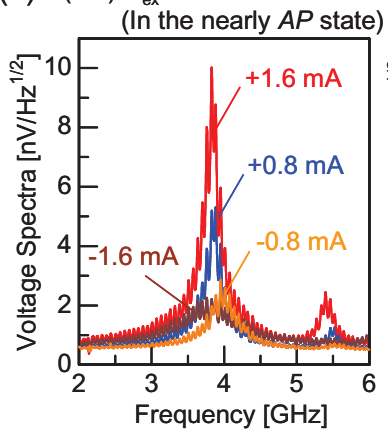

(b) (b-1) $H_{\mathrm{ex}}=+79.6 \mathrm{kA} / \mathrm{m}$

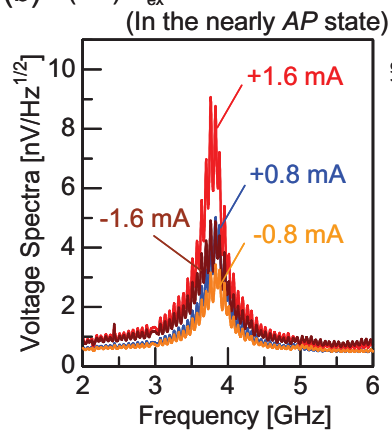

(a-2) $H_{\mathrm{ex}}=-79.6 \mathrm{kA} / \mathrm{m}$

(In the nearly $P$ state)

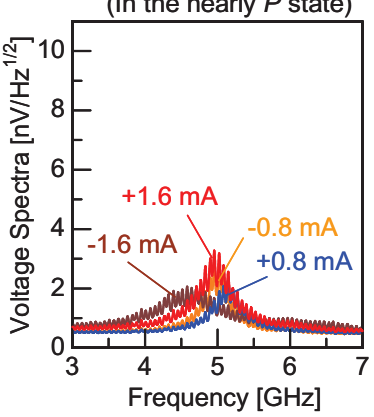

(b-2) $H_{\text {ex }}=-79.6 \mathrm{kA} / \mathrm{m}$

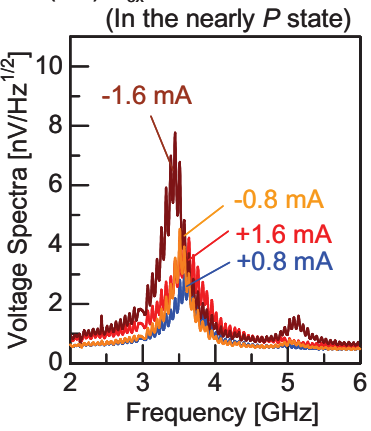

Fig. 3 Noise spectrum in TMR heads (a) \#1 and (b) \#2 as a function of dc bias current with the external magnetic field $\left(H_{\mathrm{ex}}\right)$ of $\pm 79.6 \mathrm{kA} / \mathrm{m}$. $A P$ (or $P$ ) state means that the magnetization of the free layer and that of the reference layer are nearly anti-parallel (or parallel) to each other.

polarization decreases under the negative bias current in the $P$ state.

In the case of the head \#2[Fig. 3(b-1)], at the external magnetic field of $+79.6 \mathrm{kA} / \mathrm{m}$ which results in the nearly $A P$ state (indicated by the inset $R-H$ curve of Fig. 2(a)), a main peak at approximately $3.8 \mathrm{GHz}$ becomes sharper and its intensity is markedly enhanced when the bias current direction is changed from negative to positive and its amplitude is increased. On the other hand, as can be seen in Fig. 3(b-2), an opposite trend is observed at the external magnetic field of -79.6 $\mathrm{kA} / \mathrm{m}$ which results in the nearly $P$ state. Thus, the noise originated from the magnetization fluctuation is either enhanced or reduced depending on the bias current direction, as was reported in ref. 11). For the positive bias current, ST influences the magnetization fluctuation in the $A P$ state. In this case, the magnetization fluctuation is reduced in the $P$ state and enhanced in the $A P$ state. For the negative bias current, ST influences the magnetization fluctuation in the $P$ state. The effect of ST on the magnetization fluctuation is reversed compared to the positive bias current case. On the basis of these results, it is obvious that the thermal mag-noise is influenced by the ST under the positive (or negative) bias current in the case of the nearly $A P$ (or $P$ ) state.

Furthermore, in order to clarify the influence of the ST on the noise spectrum of these heads, the frequency $(f)$, the linewidth $(\Delta f)$ and the peak intensity/current

(a) TMR head \#1
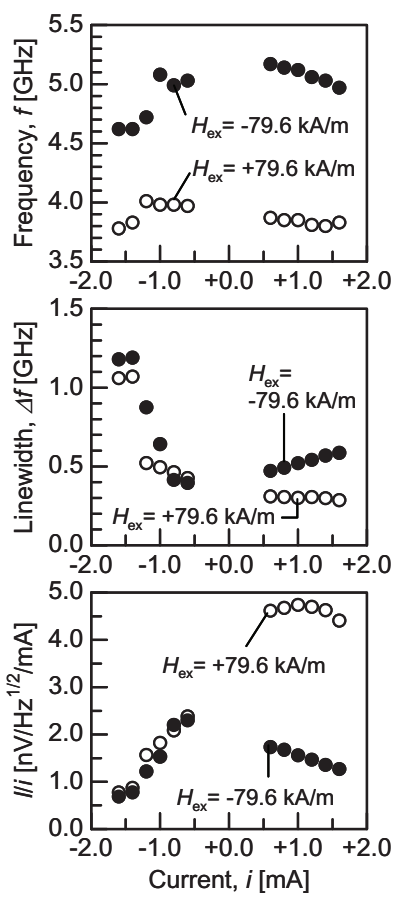

Fig. 4 Change in the frequency $(f)$, linewidth $(\Delta f)$, and peak intensity/current amplitude $(I / i)$ of the main peak for the TMR head (a) \#1 and (b) \#2 with the dc bias current. The external magnetic field of \pm 79.6 $\mathrm{kA} / \mathrm{m}$ is applied in parallel to the head plane.

amplitude $(I / l)$ of the main peak in the noise spectrum are summarized as a function of dc bias current with applied external magnetic field of $\pm 79.6 \mathrm{kA} / \mathrm{m}$. Their results are shown in Fig. 4 . In the case that the external magnetic field of $+79.6 \mathrm{kA} / \mathrm{m}$ is applied to the head \#1(in the nearly $A P$ state) [Fig. 4(a)], the main spectral-peak frequency lowers as the current intensifies regardless of the current direction. This behavior is slightly asymmetric. The linewidth slightly decreases (or markedly increases) when the bias current amplitude is increased in the positive (or negative) bias current range. Furthermore, for the positive bias current, the peak intensity/current amplitude slightly increases until the current amplitude approaches $1.0 \mathrm{~mA}$, and then slightly decreases in the range of between 1.2 and $1.6 \mathrm{~mA}$. When the bias current direction is reversed and its amplitude is increased, the peak intensity/current amplitude monotonically decreases. On the other hand, in the case that the external magnetic field of $-79.6 \mathrm{kA} / \mathrm{m}$ is applied (in the nearly $P$ state), the main spectral-peak frequentcy lowers as the current intensifies regardless of the current direction. This behavior is asymmetric. The linewidth monotonically increases (or markedly increases) in the positive (or negateve) bias current range. The peak intensity/current amplitude monotonically decreases with increasing the bias current amplitude regardless of the bias current direction. From these results, it can be summarized that the noise spectrum is 
influenced by the ST only when the bias current is positive and its amplitude is more than $1.0 \mathrm{~mA}(1.0 \times$ $\left.10^{7} \mathrm{~A} / \mathrm{cm}^{2}\right)$ in the case of nearly $A P$ state. This current density is lower than those in the CPP-GMR head ${ }^{8)}$ and the TMR head ${ }^{9)}$, and is similar to that in the magnetic tunnel junction with $20 \% \mathrm{MR}$ ratio developed for readhead applications ${ }^{10)}$. It is also noted that the RA of our TMR heads is higher than those in the CPP-GMR head $^{8)}$ and the TMR head ${ }^{9)}$. These results suggest that the ST should influence the magnetization fluctuation at the bias current density below the critical value in the case of the TMR head with high RA.

In the case that the external magnetic field of +79.6 $\mathrm{kA} / \mathrm{m}$ is applied to the head \#2(in the nearly $A P$ state) [Fig. 4(b)], the main spectral-peak frequency slightly lowers as the current intensifies regardless of the current direction. The linewidth slightly increases (or markedly increases) when the bias current amplitude is increased in the positive (or negative) bias current range. Furthermore, for the positive bias current, the peak intensity/current amplitude slightly increases until the current amplitude approaches $1.0 \mathrm{~mA}$, and then slightly decreases in the range of between 1.2 and $1.6 \mathrm{~mA}$. When the bias current direction is reversed and its amplitude is increased, the peak intensity/current amplitude monotonically decreases. On the other hand, in the case that the external magnetic field of -79.6 $\mathrm{kA} / \mathrm{m}$ is applied (in the nearly $P$ state), the main spectral-peak frequency slightly lowers as the current intensifies regardless of the current direction. This behavior is slightly asymmetric. The changes in the linewidth and the peak intensity/current amplitude with the bias current are complete opposite of those in the positive direction of external magnetic field. Thus, these results demonstrate that the effect of the ST on the noise spectrum appears under the positive (or negative) bias current in the case of nearly $A P$ (or $P$ ) state, as was reported in ref. 9, 11). Therefore, it is found that the ST play an important role with the enhancement of the thermal mag-noise originated from the free layer under the positive (or negative) bias current in the case of nearly $A P$ (or $P$ ) state.

\section{Conclusion}

We have studied noise spectra in the $\mathrm{GHz}$ range of TMR read heads and observed several magnetic noises originated from the magnetization fluctuation. A strong noise spectral peak at around $3-4 \mathrm{GHz}$ is either enhanced or reduced depending on both the bias current and the external magnetic field. The reason for this is the effect of the ST on the thermally excited magnetization fluctuation (thermal mag-noise) originated from the free layers. Other weak noises are mainly reduced with increasing external magnetic field intensity or are almost constant regardless of external magnetic field. These noises are attributed by either only the thermal mag-noise or the non-uniform magnetization fluctuation at the edge of the free layer. From these results, it can be seen that the ST influences the enhancement of magnetic noise under the positive (or negative) bias current in case the magnetization of the free and reference layers are nearly anti-parallel (or parallel) to each other.

Acknowledgements This work has been supported in part by Storage Research Consortium (SRC).

\section{References}

1) S. Mao, E. Linville, J. Nowak, Z. Zhang, S. Chen, B. Karr, P. Anderson, M. Ostrowski, T. Boonstra, H. Cho, O. Heinonen, M. Kief, S. Xue, J. Price, A. Shukh, N. Amin, P. Kolbo, P.-L. Lu, P. Steiner, Y. C. Feng, N.-H. Yen, B. Swanson, and P. Ryan: IEEE Trans. Magn., 40, 307 (2004).

2) K. Hoshino, H. Hoshiya, H. Katada, N. Yoshida, K. Watanabe, and K. Nakamoto: IEEE Trans. Magn., 41, 2926 (2005).

3) H. Oshima, K. Nagasaka, A. Jogo, T. Ibusuki, Y. Shimizu, and A. Tanaka: IEEE Trans. Magn., 41, 2929 (2005).

4) N. Smith and P. Arnett: Appl. Phys. Lett., 78, 1448 (2001).

5) J.-G. Zhu: J. Appl. Phys., 91, 7273 (2002).

6) O. Heinonen and H. S. Cho: IEEE Trans. Magn., 40, 2227 (2004).

7) J.-G. Zhu and X. Zhu: IEEE Trans. Magn., 40, 182 (2004).

8) N. Smith, J. A. Katine, J. R. Childress, and M. J. Carey: IEEE Trans. Magn., 42, 114 (2006).

9) J.-G. Zhu, Y. Zhou, and S. Mao: IEEE Trans. Magn., 42, 2441 (2006).

10) H. Katada, K. Nakamoto, H. Hoshiya, K. Hoshino, and N. Yoshida: IEEE Trans. Magn., 42, 2450 (2006).

11) S. Petit, C. Baraduc, C. Thirion, U. Ebels, Y. Liu, M. Li, P. Wang, and B. Dieny: Phys. Rev. Lett., 98, 077203-1 (2007).

12) H. N. Bertram, V. L. Safonov, and Z. Jin: IEEE Trans. Magn., 38, 2514 (2002).

Received May. 25, 2009; Revised July. 15, 2009; Accepted Sep. 13,2009 